\title{
Atmospheric quasi-14 month fluctuation and excitation of the Chandler wobble
}

\author{
Yuichi Aoyama ${ }^{1}$, Isao Naito ${ }^{2}$, Tetsuya Iwabuchi ${ }^{3 *}$, and Nobuo Yamazaki ${ }^{3}$ \\ ${ }^{1}$ Radio Science Center for Space and Atmosphere, Kyoto University, Uji, Japan \\ ${ }^{2}$ National Astronomical Observatory/Graduate University for Advanced Studies, Tokyo, Japan \\ ${ }^{3}$ Meteorological Research Institute, Tsukuba, Japan \\ (Received November 18, 2003; Revised December 26, 2003; Accepted December 26, 2003)
}

\begin{abstract}
A quasi-14 month fluctuation in the atmospheric excitation function for the Earth's wobble is discussed by using the re-analysis data of the European Center for Medium-range Weather Forecast for the 14 years between 1980 and 1993. The spectrum of the atmospheric wind excitation function shows a striking peak near the 14-month period. As a result, the atmospheric (wind plus pressure) excitation function shows exactly the same power as that of the geodetic excitation function inferred from the observed wobble at the Chandler wobble frequency (about 14 months), suggesting that the atmosphere excited the Chandler wobble between 1980 and 1993. The wind fluctuation comes mostly from the tropospheric wind.
\end{abstract}

Key words: ECMWF wind, quasi-14-month fluctuation, atmospheric excitation, Chandler wobble.

\section{Introduction}

The Chandler wobble $(\mathrm{CW})$ is a free oscillation of the Earth's polar motion with about a 14-month period (Munk and MacDonald, 1960; Lambeck, 1980). Ceaseless free oscillations need excitations and so does CW that has exhibited various dynamical aspects during its history of over a hundred years (e.g., Lambeck, 1980). Despite the fact that its excitation processes inside and outside the Earth's mantle have been proposed, no major source has yet been identified. Lately, however, variations in the atmosphere and ocean have been widely noticed as a possible source (Wahr, 1983; Furuya et al., 1996; Plag, 1997; Celaya et al., 1999; Ponte and Stammer, 1999; Gross, 2000; Aoyama and Naito, 2001; Gross et al., 2003).

Among these researchers, Gross (2000) has successfully elucidated ocean bottom pressure fluctuations to be the major excitation source for the years 1985-1996. In addition to the atmospheric excitation functions computed from the reanalysis data of the National Center for Environmental Prediction (NCEP), he used the oceanic excitation functions of Ponte et al. (1998), in which the ocean current and bottom pressure contributions to the wobble were computed from the product of a global oceanic general circulation model (OGCM) which was driven by the wind-stress fields, surface heat and freshwater flux fields of the NCEP re-analysis data. Here he regarded the atmospheric wind as playing a minor role in the $\mathrm{CW}$ excitation.

On the other hand, Aoyama and Naito (2001) have shown that near a 14-month period, the spectrum of the atmospheric wind excitation function has a striking peak and that the

*Now at University Coorperation for Atmospheric Research, Boulder, USA.

Copy right(c) The Society of Geomagnetism and Earth, Planetary and Space Sciences (SGEPSS); The Seismological Society of Japan; The Volcanological Society of Japan; The Geodetic Society of Japan; The Japanese Society for Planetary Sciences. spectral power of the atmospheric (wind plus pressure) excitation function is exactly the same as that of the geodetic excitation function inferred from the observed wobble at the CW frequency for the years 1983-1998. Here, the data used was the atmospheric excitation function computed from the operational analysis data of the Japan Meteorological Agency (JMA). Moreover, they found that the wind excitation function from the NCEP only makes a minor contribution to $\mathrm{CW}$ as well as there being no quasi-14 month periodicity.

Since the above two studies conflict with each other on a point of wind behavior near the $\mathrm{CW}$ frequency, we need a third atmospheric data set to reconfirm the wind behavior. In order to do this, we can use the re-analysis data of the European Center for Medium-range Weather Forecast (ECMWF). In addition, no study of the atmospheric excitation of $\mathrm{CW}$ has been reported so far using the ECMWF re-analysis data, despite the fact that the ECMWF operational analysis data once played a key role in identifying atmospheric excitations of the Earth's high precision rotations determined by space geodetic techniques (e.g., Dickey, 1993). The present paper revisits the atmospheric (wind plus pressure) excitation for $\mathrm{CW}$ by using the ECMWF re-analysis data with emphasis on the quasi-14 month fluctuation of the atmospheric wind excitation. The results of the analysis reveal that the wind has a quasi-14 month periodicity which was thought to excite CW during the period of 1980-1993.

\section{Dynamics, Data and Analysis}

The wobble is a variation in orientation of the instantaneous angular velocity vector of the mantle with respect to the Earth's figure axis. Under the conservation of the Earth's total angular momentum in the absence of external torques, the wobble is caused by exchanges of the equatorial angular momentum between the mantle and the geophysical fluids. The dynamics is described by the linearized Liouville equa- 

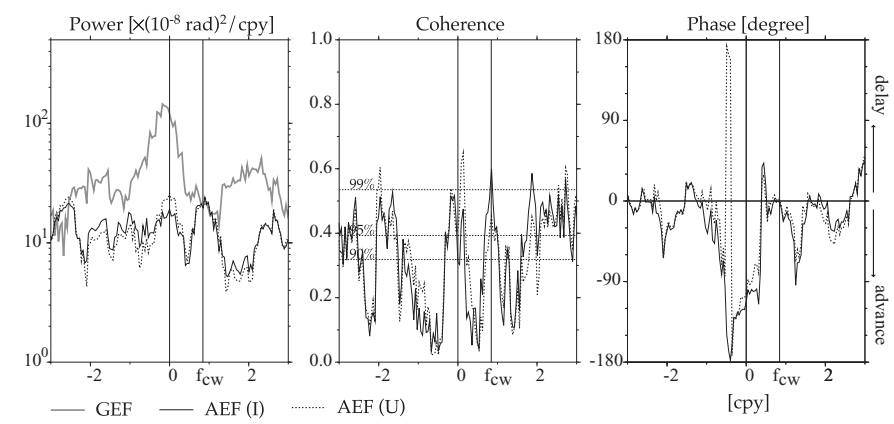

Fig. 1. Power spectra, squared coherence, and phase difference of/between the geodetic excitation function (GEF) and the atmospheric (wind plus IB-pressure) excitation functions (AEF) from the initialized (I) and uninitialized (U) data at frequency bands from -3 to 3 cpy for the 14 years of $1980-1993$, where $f_{\mathrm{cW}}=0.847$ cpy and $Q_{\mathrm{cw}}=179$ are assumed.

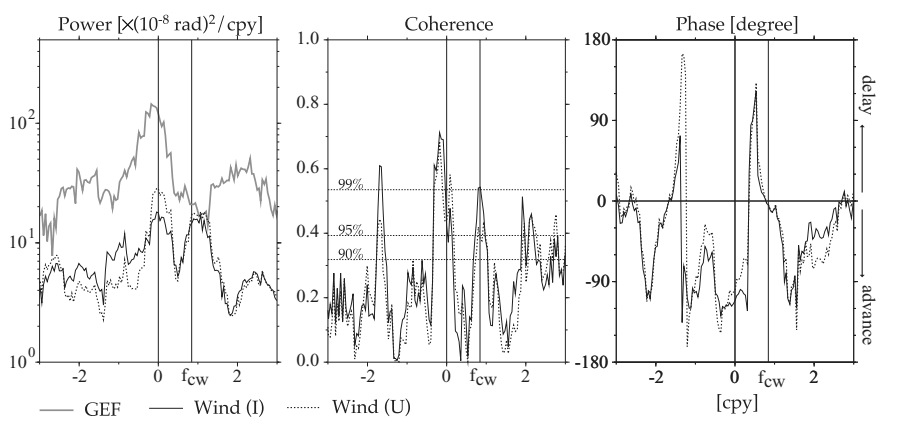

Fig. 2. The same as Fig. 1, but for the wind term.

tion

$$
\tilde{m}+\left(i / \tilde{\sigma}_{\mathrm{cw}}\right) \mathrm{d} \tilde{m} / \mathrm{d} t=\tilde{\chi}
$$

where $\tilde{m}$ is dimensionless equatorial angular velocity vector, $\tilde{\sigma}_{\mathrm{cw}}=2 \pi f_{\mathrm{cw}}\left(1+i / 2 Q_{\mathrm{cw}}\right)$ is the complex CW frequency including the dissipation with the quality factor $Q_{\mathrm{cw}}$ at the $\mathrm{CW}$ frequency $f_{\mathrm{cw}}$, and $\tilde{\chi}$ is the excitation function representing dimensionless inertia products and changes in the relative angular momentum of the geophysical fluids that can be evaluated from atmospheric pressure, wind, ocean current, ocean bottom pressure, land water storage, etc. (Munk and MacDonald, 1960; Lambeck, 1980). In general, the left side of Eq. (1) is defined as the geodetic excitation function because it depends only upon the geodetically determined $\tilde{m}$. In the following analysis, we discuss the atmospheric excitation for CW using an excitation domain approach that compares the atmospheric excitation function with the geodetic one in the frequency domain.

The geodetic excitation function is computed from the observed wobble data in SPACE98 (Gross et al., 1998), by using the discrete form of equation (2a) of Wilson (1985), where $f_{\mathrm{cw}}=0.847$ cycle per year (cpy) and $Q_{\mathrm{cw}}=179$ (Wilson and Vicente, 1990) are employed. On the other hand, the atmospheric excitation function consists of the wind and pressure terms that reflect, respectively, the effects of the relative angular momentum due to wind and of the inertia products due to surface-pressure variation. The pressure term here is based upon the inverted barometer (IB) approximation that assumes an isostatic response of the ocean to the atmospheric pressure loading, since the dynamics is discussed on a time scale longer than three months. The transfer functions of 1.098 for the pressure term and 1.5913 for the wind term are used in correcting the yielding of the mantle and decoupling of the core (see equations (2) and (3) of Aoyama and Naito (2001), for details).

For computing the atmospheric excitation function, initialized and uninitialized data sets are used from the 15year re-analysis of ECMWF ERA-15 (Gibson et al., 1997). In general, both reflect more closely the modeled and observed data, respectively. Since the NCEP reanalysis and the JMA operational analysis data, respectively, are initialized and uninitialized data sets, we can consider that there were some differences in the wind behavior due to the initialization between the two data sets. Therefore, we can detect the effects of the initialization on the atmospheric wind excitation to $\mathrm{CW}$ by using the two above data sets of ECMWF. The data assimilation system used for ERA-15 is a spectral T106 resolution forecast model with 31 vertical hybrid levels and intermittent statistical (optimum interpolation) analysis system with 6 hour cycling. The principal source of the observations is the ECMWF real-time data collection.

In analysis, we compute power spectra, squared coherence and phase difference of and between the geodetic and atmospheric excitation functions, using the multitaper method (Thomson, 1982) (see Aoyama and Naito (2001) for details). All seasonal and linear terms are removed by least square fitting before analysis. The analysis period is taken for the 14 years between 1980 and 1993.

\section{Atmospheric Quasi-14 Month Fluctuation Ex- citing the Chandler Wobble}

Figure 1 shows the power spectra, squared coherence, and phase difference of and between the geodetic excitation function (hereafter GEF) and the atmospheric wind plus 

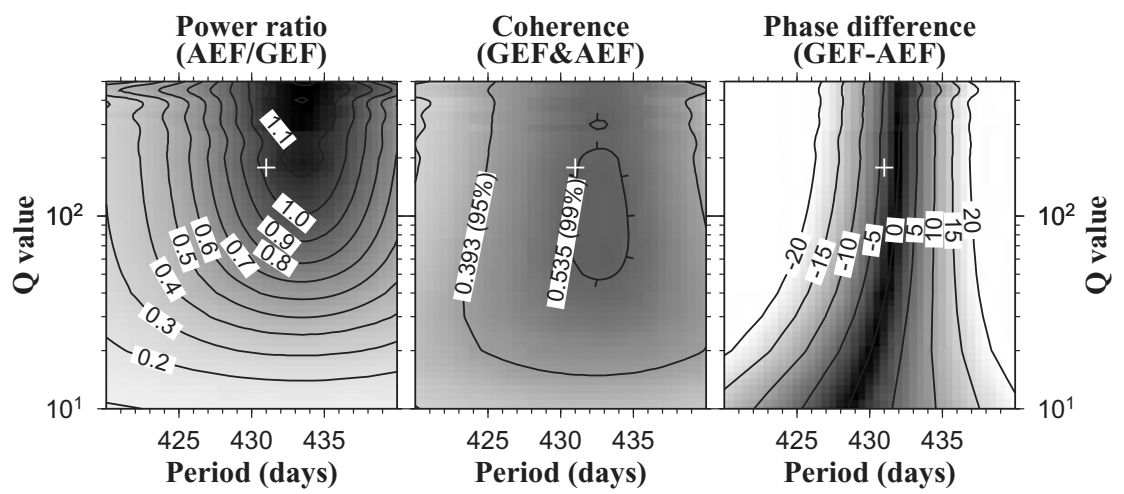

Fig. 3. Dependencies of excitation power ratio, squared coherence, and phase difference upon $f_{\mathrm{cw}}$ and $Q_{\mathrm{cw}}$ between the geodetic excitation function (GEF) and the atmospheric (wind plus IB-pressure) excitation function (AEF) from the initialized data. + denotes $f_{\mathrm{cw}}=0.847 \mathrm{cpy}$ and $Q_{\mathrm{cw}}=179$ assumed in Figs. 1 and 2, and Table 1.

IB-pressure excitation functions (hereafter AEF) from the initialized and uninitialized data at the frequency band between -3 cpy to 3 cpy. Although there exist large deficiencies in the power of AEFs compared to that of the GEF, AEFs show a striking spectral peak with exactly the same power as that of the GEF near the CW frequency (approximately 14 months), in which the coherences are high and the phase differences are close to zero. The deficient powers in AEFs are possibly supplied by oceanic variations (Gross, 2000). Although, on the other hand, there exist differences in power as well as in the shape of the spectra between the two AEFs based on the initialized and uninitialized data, they agree with each other near the $\mathrm{CW}$ frequency, indicating that there is little effect of the initialization on the atmospheric excitation for $\mathrm{CW}$. In other words, it is suggested that the difference in the wind contribution to $\mathrm{CW}$ based upon the NCEP and JMA data sets demonstrated by Aoyama and Naito (2001) was not caused by the initializations. Therefore the atmospheric quasi-14-month fluctuations exciting $\mathrm{CW}$ appeared in both ECMWF re-analysis data sets during the period of 1980-1993 and the JMA operational analysis data during the period of 1983-1998, is considered to be real.

Figure 2 is same as Fig. 1, but only for the wind terms. It turns out that the spectral peak of AEFs near the CW frequency shown in Fig. 1 comes mostly from the wind terms. Also, the differences between the power spectra of the two AEFs shown in Fig. 1 seem to correspond to those between the two wind terms based upon the initialized and uninitialized data. In fact, we confirmed that the two IB-pressure terms from the initialized and uninitialized data show nearly same spectra over the frequency band from -3 cpy to 3 cpy. Table 1 shows the excitation powers, squared coherences, and phase differences near the $\mathrm{CW}$ frequency of and between the geodetic excitation function and the wind plus IBpressure, wind, IB-pressure, tropospheric (surface-100 hPa) wind, and stratospheric $(100-10 \mathrm{hPa})$ wind terms, where the numerical values are integrated over the frequency band of $0.8-0.9$ cpy (there are three points of $0.803,0.847$, and 0.892 cpy in this band due to the short data length). It turns out that the IB-pressure terms during the period of 1980-1993 make only very small contributions to $\mathrm{CW}$ and that the major atmospheric contribution comes from the wind, in particular, from the tropospheric wind.
Table 1. Excitation powers, coherences, and phase differences.

\begin{tabular}{lcccr}
\hline & \multicolumn{2}{c}{$\begin{array}{c}\text { Power } \\
\left(10^{-8} \mathrm{rad}\right)^{2}\end{array}$} & Coherence & $\begin{array}{c}\text { Phase } \\
\text { (degree) }\end{array}$ \\
\hline GEF & & 2.80 & - & - \\
AEF & $\mathrm{I}$ & 2.88 & 0.60 & -1.9 \\
& $\mathrm{U}$ & 2.96 & 0.45 & 1.0 \\
Wind & $\mathrm{I}$ & 1.98 & 0.54 & -5.1 \\
& $\mathrm{U}$ & 2.22 & 0.38 & -4.5 \\
IB-pressure & $\mathrm{I}$ & 0.75 & 0.07 & 12.8 \\
& $\mathrm{U}$ & 0.78 & 0.06 & 26.0 \\
Tropospheric wind & $\mathrm{I}$ & 1.90 & 0.64 & -3.3 \\
& $\mathrm{U}$ & 2.01 & 0.49 & -3.7 \\
Stratospheric wind & $\mathrm{I}$ & 0.16 & 0.03 & -157.1 \\
& $\mathrm{U}$ & 0.16 & 0.03 & -172.0 \\
\hline
\end{tabular}

Excitation powers, squared coherences, and phase differences of and between the geodetic excitation function and the wind plus IBpressure, wind, and IB-pressure, tropospheric (surface-100 hPa) wind, and stratospheric $(100-10 \mathrm{hPa})$ wind terms integrated over the $\mathrm{CW}$ frequency band, where $f_{\mathrm{cw}}=0.847$ cpy and $Q_{\mathrm{cw}}=179$ are assumed. I and $U$ denote those from the initialized and uninitialized data, respectively.

\section{Discussion and Summary}

The above results are obtained by assuming $f_{\mathrm{cw}}=0.847$ cpy and $Q_{\mathrm{cw}}=179$ estimated from 86 years data of the observed polar motion by Wilson and Vicente (1990). In general, statistical estimates of the parameters of $f_{\mathrm{cw}}$ and $Q_{\mathrm{cw}}$ not only depend upon data length but also on data precision. In addition, the estimated parameters affect the spectral structure of the geodetic excitation function. As a result, the excitation efficiency for $\mathrm{CW}$ depends strongly upon the atmospheric excitation function with a spectral peak near the $\mathrm{CW}$ frequency. Therefore, we need to check whether or not these parameters match the $\mathrm{CW}$ excitation results obtained above. Figure 3 shows the dependencies of the excitation power ratio, squared coherence, and phase difference between GEF and AEF upon $f_{\mathrm{cw}}$ and $Q_{\mathrm{cw}}$, where the AEF is computed from the initialized data. It turns out that the previously assumed parameters $f_{\mathrm{cw}}=0.847$ cpy and $Q_{\mathrm{cw}}=179$, denoted by + in the figure, are roughly appropriate, even for this analysis with a short data length.

A small spectral peak in the wind excitation similar to that in Fig. 2 was found in the case of the JMA operational analy- 
sis data by Aoyama and Naito (2001) (see also Furuya et al., 1996). As previously mentioned, however, they found no peak in the case of the NCEP re-analysis data. In general, the spectral structures of the atmospheric wind excitation functions from the ECMWF and JMA data are very close to each other, in particular near the CW frequency, but they are largely different from that of the NCEP (Aoyama and Naito, 2001). The integrated excitation power near the CW frequency of the wind contribution from the JMA data, for example, shows $2.27\left(10^{-8} \mathrm{rad}\right)^{2}$, while that from the NCEP data shows $0.31\left(10^{-8} \mathrm{rad}\right)^{2}$. This discrepancy is a weak point to argue the atmospheric excitation to $\mathrm{CW}$, but a similar problem can be involved in the oceanic $\mathrm{CW}$ excitation proposed by Gross (2000), because he used the ocean current and bottom pressure contributions to the wobble based upon the OGCM data driven by the wind stress fields of the NCEP re-analysis data that possibly differ in part from those of the JMA and ECMWF analysis data. It should be noticed that the latest atmospheric general circulation models (AGCM), in general, reasonably simulate the axial angular momentum variations (e.g., Hide et al., 1997) but not the equatorial ones (Naito et al., 2000) in comparison with the angular momentum variations inferred from the observed rotation of the Earth. In addition, the simulation study based on AGCM demonstrated that the atmospheric excitation to CW shows strong time variability (Celaya et al., 1999).

On the other hand, the quasi-14-month fluctuation that appeared in the wind contribution is a very interesting phenomenon, possible not only connected with the wind forced pole tide in the North Sea (e.g., Tsimplis et al., 1994; O'Connor et al., 2000) but also with a 14-month oceanic variation in the equatorial Pacific (e.g., Naito, 1983; Philander et al., 1989). Concerning this, Plag (1997) presented an interesting hypothesis that the observed $\mathrm{CW}$ and its pole tide at sea level were forced by the 14-16-month atmospheric pressure fluctuations related to the climate system. However, we must wait for precise descriptions of its mechanism maintaining the $\mathrm{CW}$ excitation until the re-analysis data from a more advanced data assimilation system with a more realistic AGCM, become available for analyzing this type of very small amplitude low frequency variations in the atmosphere.

In summary, although the ocean-bottom pressure fluctuation has been considered to be the most important source exciting $\mathrm{CW}$, the atmospheric excitation is discussed by using the ECMWF reanalysis data sets for the 14 years between 1980 and 1993. The results of analysis reveal that the quasi-14-month fluctuation of the atmospheric wind plays an important role in the $\mathrm{CW}$ excitation, at least for the analysis period. Its power arised mainly from the tropospheric wind with about a 14-month periodicity. However, there exist large spectral gaps between the geodetic and atmospheric excitation power spectra, except near the $\mathrm{CW}$ frequency, that should be filled up by other sources such as oceanic variations forced by the winds. Therefore, it may be concluded that each of the main comparable sources (atmospheric pressure, wind, and ocean-bottom pressure) intermittently and in turn play a dominant role in exciting $\mathrm{CW}$.

Acknowledgments. The authors thank H-P. Plag and C. R. Wilson for valuable comments on this paper.

\section{References}

Aoyama, Y. and I. Naito, Wind contributions to the Earths angular momentum budgets in seasonal variation, J. Geophys. Res., 105, 12417-12431, 2000.

Aoyama, Y. and I. Naito, Atmospheric excitation of the Chandler wobble, 1983-1998, J. Geophys. Res., 106, 8941-8954, 2001.

Celaya, M. A., J. M. Wahr, and F. O. Bryan, Climate-driven polar motion, J. Geophys. Res., 104, 12813-12829, 1999.

Dickey, J. O., Atmospheric excitation of the Earths rotation: Progress and prospects via space geodesy, in Contributions of Space Geodesy to Geodynamics: Earth Dynamics, Geodynamics Ser, vol. 24, edited by D. E. Smith and D. L. Turcotte, pp. 55-70, AGU, Washington, D. C., 1993.

Eubanks, T. M., Variations in the orientation of the Earth, in Contributions of Space Geodesy to Geodynamics: Earth Dynamics, Geodynamics Ser. vol. 24, edited by D. E. Smith and D. L. Turcotte, pp.1-54, AGU, Washington, D. C., 1993.

Furuya, M., Y. Hamano, and I. Naito, Quasi-periodic wind signal as a possible excitation of Chandler wobble, J. Geophys. Res., 101, 25537 25546, 1996.

Gibson, J. K., P. Kallberg, S. Uppala, A. Hernandez, A. Nomura, and E. Serrano, ERA Description, Re-Analysis (ERA) Project Report Series 1, ECMWF, Shinfield Park, Reading, England, 72 pp., 1997.

Gross, R. S., T. M. Eubanks, J. A. Steppe, A. P. Freedman, J. O. Dickey, and T. F. Runge, A Kalman-filter-based approach to combining independent Earth-orientation series, J. Geodesy, 72, 215-235, 1998

Gross, R. S., The excitation of the Chandler wobble, Geophys. Res. Lett. 27, 2329-2332, 2000.

Gross, R. S., I. Fukumori, and D. Menemenlis, Atmospheric and oceanic excitation of the Earth's wobbles during 1980-2000, J. Geophys. Res., 108, 2370, doi:10.1029/2002JB002143, 2003.

Hide, R., J. O. Dickey, S. L. Markus, R. D. Rosen, and D. A. Salstein, Atmospheric angular momentum fluctuations during 1979-1988 simulated by global circulation models, J. Geophys. Res., 102, 16423-16438, 1997.

Lambeck, K., The Earth's Variable Rotation, 449 pp., Cambridge Univ. Press, New York, 1980.

Munk, W. H. and G. J. F. MacDonald, The Earth Rotation, 323 pp., Cambridge Unv. Press, New York, 1960.

Naito, I., Response of the Ocean to the Chandler Wobble, Marine Geodesy, 7, 345-358, 1983

Naito, I., Y.-H. Zhou, M. Sugi, R. Kawamura, and N. Sato, Threedimensional atmospheric angular momentum simulated by the Japan Meteorological Agency model for the period of 1955-1994, J. Meteorol. Soc. Japan, 78, 111-122, 2000

O'Connor, W. P., B. F. Chao, D. Aheng, and A. Y. Au, Wind stres forcing of the North Sea 'Pole Tide', Geophys. J. Int., 142, 620-630, 2000.

Philander, S. G. H., N. C. Lau, R. C. Pacanowski, and M. J. Nath, Two different simulations of the Southern Oscillation and El Nino with coupled ocean-atmosphere general circulation models, Phil. Trans. R. Soc. Lond. A., 329, 167-178, 1989.

Plag, H.-P., Chandler wobble and pole tide in relation to interannual atmosphere-ocean dynamics, in Tidal Phenomena, Lecture Notes in Earth Sciences, 66, edited by H. Wilhelm, W. Zurn, and H.-G. Wenzel, pp. 183 218, Springer, Berlin, 1997.

Ponte, R. M. and D. Stammer, Role of ocean currents and bottom pressure variability on seasonal polar motion, J. Geophys. Res., 104, 2339323409, 1999.

Ponte, R. M., D. Stammer, and J. Marshall, Oceanic signals in observed motions of the Earth's pole of rotation, Nature, 391, 476-479, 1998

Thomson, D. J., Spectrum estimation and harmonic analysis, Proc. IEEE, 70, 1055-1096, 1982.

Tsimplis, M. N., R. A. Flather, and J. M. Vassie, The North Sea pole tide described through a tide-surge numerical model, Geophys. Res. Lett., 21, 449-452, 1994.

Wilson, C. R., Discrete polar motion equations, Geophys. J. R. Astron. Soc., 80, 551-554, 1985 .

Wilson, C. R. and R. O. Vicente, Maximum likelihood estimates of polar motion parameters, in Variation in Earth Rotation, Geophys. Monogr. Ser., vol. 59, edited by D. D. McCathy and W. E. Carter, pp. 151-155, AGU, Washington, D.C., 1990.

Y. Aoyama (e-mail: aoyama@kurasc.kyoto-u.ac.jp), I. Naito, T. Iwabuchi, and N. Yamazaki 